\title{
Assessing the bias in blocked two level fractional factorial designs with respect to confounding pattern
}

\author{
Jamilu Garba*, Abubakar Yahaya, Hussaini Garba Dikko \\ Department of Mathematics, Ahmadu Bello University, Zaria, Nigeria \\ *Corresponding authorE-mail: gyjamil@yahoo.com
}

Copyright $\odot 2014$ Jamilu Garba et al. This is an open access article distributed under the Creative Commons Attribution License, which permits unrestricted use, distribution, and reproduction in any medium, provided the original work is properly cited.

\begin{abstract}
Blocked two-level fractional factorial designs are very useful in screening experiment and other areas of scientific research. In some experiments, apart from the main effects, some two-factor interactions may be important and should be estimated. Thus, the postulated model should include all main effects, some important two-factor interactions and blocking effects. The remaining two factor interaction and some other higher order interactions not included in the postulated model may confound and bias the estimate of the effects in the model which in turn lower the precision of the parameter estimate. It is therefore necessary to select an optimal design from the design space that will minimize this bias (contamination). In this article, two designs were selected with respect to confounding pattern and the bias accounted in the estimation of the regression coefficient of the postulated models were evaluated and compared. The confounding pattern of design I and II is $(0,6,1)$ and $(4,2,2)$ respectively; it was observed that bias accounted in the estimation of regression coefficient is higher in design II.
\end{abstract}

Keywords: Confounding Pattern, Defining Contrast Subgroup, Defining Words, Minimum Aberration, and Word-Length Pattern.

\section{Introduction}

Fractional factorial designs enable researchers to investigate many factors in an economical number of runs. In particular, for two-level factors, a regular fractional factorial design with $n$ factors and $N=2^{\text {n-k }}$ runs can accommodate up to $n=N-1$ factors for resolution III designs. Small fractional factorial designs are often performed as completely randomized designs. However the larger the run size $N$, the more advantageous running the experiment in blocks becomes [17]. Then how can we block and come up with a better selected design?

The most commonly used criterion for $2^{\mathrm{m}-\mathrm{p}}$ design selection is the minimum aberration criterion proposed by Fries and Hunter [6]. For small number of factors, Franklin [5] provided a useful catalogue of $2^{\mathrm{m}-\mathrm{p}}$ designs with minimum aberration. Several researchers addressed the construction of minimum aberration blocked designs. Sitter, et al [9] provided some collections of minimum aberration blocked designs with all 8 and 16 runs, 32 runs up to 15 factors, 64 runs up to 9 factors, and 128 runs up to 9 factors. Chen and Cheng [2] developed a theory to characterize minimum aberration blocked designs in terms of their blocked residual designs and gave collection of minimum aberration blocked designs with all 8 and 16 runs, and 32 runs up to 20 factors. Cheng and $\mathrm{Wu}$ [4] compared minimum aberration blocked designs with respect to different combined word length patterns for $8,16,32,64$ and128 runs up to 9 factors; they also provide collections of minimum aberration blocked designs with all 27 runs, and 81 runs up to 10 factors. Xu [15] and $\mathrm{Xu}$ and Lau [16] further developed some theories and constructed minimum aberration blocked designs with all 32 runs, all 81 runs, and 64 runs up to 32 factors.

All these researchers discussed blocking schemes for the models containing main effects and blocking effects only. Their discussions were mainly based on the schemes that have optimal estimation of the main effects and the blocking effects of the experiments. However, Ke [14] considered the selection of blocked $2^{\mathrm{m}-\mathrm{p}}$ designs when some two factor interactions are considered to be important. When some

2 -factor interactions are important; the postulated model should consist of all main effects, blocking effect and these important 2-factor interactions. If the effects not in the postulated model cannot be completely ignored, they bias the 
estimate of the effects in the model. To solve this problem, the key issues are to permit estimation of the main effects, blocking effects, and the important 2-factor interactions in the postulated model and then minimize the bias caused by the other effects not included in the postulated model [14]. To minimize this bias, an optimal design should be selected such that the contamination caused by these non-negligible effects is minimized. This can be achieved by sequentially minimizing the confounding pattern of the design [14]. In this article we evaluate the negative effect of this contamination on the precision of the parameter estimate.

The organization of this article is as follows: In section two the concept of two level fractional factorial designs was discussed. The criterion for selecting blocked $2^{\mathrm{m}-\mathrm{p}}$ designs was presented in section three. In section four the performance of designs were evaluated and a conclusion remark was offered in section five.

\section{Two level fractional factorial design}

A regular two-level fractional factorial design is commonly referred to as a $2^{\mathrm{m}-\mathrm{p}}$ design. It has $m$ two-level factors with $2^{\mathrm{m}-\mathrm{p}}$ runs, and commonly determined by $p$ independent defining relations. When $p=0$, a $2^{\mathrm{m}-\mathrm{p}}$ design reduces to a full factorial of a $2^{\mathrm{m}}$ design. A defining relation is given by a word of letters which are levels of the factors denoted by 1,2 . . . $m$. The number of letters in a word is called its word length. The group of defining words generated by $p$ independent defining words is called the defining contrast subgroup of the design. The length of the shortest word in the defining contrast subgroup is called the resolution of a design. The vector $W(\mathrm{~d})=\left[A_{1}(\mathrm{~d}), A_{2}(\mathrm{~d}), \ldots, A_{\mathrm{m}}(\mathrm{d})\right]$ is called the word length pattern of the design $\mathrm{d}$, where $A_{\mathrm{i}}(\mathrm{d})$ is the number of words of length $i$ in the defining contrast subgroup. The resolution of the design is the smallest $r$ satisfying $A_{r} \geq 1$. The resolution criterion proposed by Box and Hunter [1] select $2^{\mathrm{m}-\mathrm{p}}$ design that has higher resolution. Since two designs having the same resolution may have different word length pattern and may not be equally good; Fries and Hunter [6] proposed the minimum aberration criterion for further discrimination of $2^{\mathrm{m}-\mathrm{p}}$ designs. For instance consider two different designs $\mathrm{d}_{1}$ and $\mathrm{d}_{2}$, and suppose $r$ is the smallest value such that $A_{r}\left(\mathrm{~d}_{1}\right) \neq A_{r}\left(\mathrm{~d}_{2}\right)$. $\mathrm{d}_{1}$ is said to have less aberration than $\mathrm{d}_{2}$ if $A_{r}\left(\mathrm{~d}_{1}\right)<A_{r}\left(\mathrm{~d}_{2}\right)$. If no any other design has less aberration than $d_{1}$, then $d_{1}$ is said to have minimum aberration. The minimum aberration criterion which selects $2^{\mathrm{m}-\mathrm{p}}$ designs with minimum aberration is most commonly used in selecting $2^{\mathrm{m}-\mathrm{p}}$ designs. In what follows, an example was used to illustrate $2^{\mathrm{m}-\mathrm{p}}$ designs and minimum aberration. Suppose it is desired to conduct an experiment with sixteen runs and several factors at two levels, levelled +1 and -1 . Table 1 below gives the column of a sixteen runs saturated design with its columns arranged in Yates's order, with the generating independent columns 1, 2, 4 and 8 in boldface.

Table 1: Columns of a16-run saturated design in Yates's order

\begin{tabular}{|c|c|c|c|c|c|c|c|c|c|c|c|c|c|c|c|}
\hline (1) & (2) & (3) & (4) & (5) & (6) & $(7)$ & $(8)$ & (9) & $(10)$ & (11) & $(12)$ & (13) & (14) & $(15)$ & Response \\
\hline 1 & 2 & 12 & 4 & 14 & 24 & 124 & 8 & 18 & 28 & 128 & 48 & 148 & 248 & 1248 & \\
\hline-1 & -1 & 1 & -1 & 1 & 1 & -1 & -1 & 1 & 1 & -1 & 1 & -1 & -1 & 1 & $\mathrm{y}_{1}$ \\
\hline 1 & -1 & -1 & -1 & -1 & 1 & 1 & -1 & -1 & 1 & 1 & 1 & 1 & -1 & -1 & $\mathrm{y}_{2}$ \\
\hline-1 & 1 & -1 & -1 & 1 & -1 & 1 & -1 & 1 & -1 & 1 & 1 & -1 & 1 & -1 & $\mathrm{y}_{3}$ \\
\hline 1 & 1 & 1 & -1 & -1 & -1 & -1 & -1 & -1 & -1 & -1 & 1 & 1 & 1 & 1 & $\mathrm{y}_{4}$ \\
\hline-1 & -1 & 1 & 1 & -1 & -1 & 1 & -1 & 1 & 1 & -1 & -1 & 1 & 1 & -1 & $\mathrm{y}_{5}$ \\
\hline 1 & -1 & -1 & 1 & 1 & -1 & -1 & -1 & -1 & 1 & 1 & -1 & -1 & 1 & 1 & $\mathrm{y}_{6}$ \\
\hline-1 & 1 & -1 & 1 & -1 & 1 & -1 & -1 & 1 & -1 & 1 & -1 & 1 & -1 & 1 & $\mathrm{y}_{7}$ \\
\hline 1 & 1 & 1 & 1 & 1 & 1 & 1 & -1 & -1 & -1 & -1 & -1 & -1 & -1 & -1 & $\mathrm{y}_{8}$ \\
\hline-1 & -1 & 1 & -1 & 1 & 1 & -1 & 1 & -1 & -1 & 1 & -1 & 1 & 1 & -1 & $\mathrm{y}_{9}$ \\
\hline 1 & -1 & -1 & -1 & -1 & 1 & 1 & 1 & 1 & -1 & -1 & -1 & -1 & 1 & 1 & $\mathrm{y}_{10}$ \\
\hline-1 & 1 & -1 & -1 & 1 & -1 & 1 & 1 & -1 & 1 & -1 & -1 & 1 & -1 & 1 & $\mathrm{y}_{11}$ \\
\hline 1 & 1 & 1 & -1 & -1 & -1 & -1 & 1 & 1 & 1 & 1 & -1 & -1 & -1 & -1 & $\mathrm{y}_{12}$ \\
\hline-1 & -1 & 1 & 1 & -1 & -1 & 1 & 1 & -1 & -1 & 1 & 1 & -1 & -1 & 1 & $\mathrm{y}_{13}$ \\
\hline 1 & -1 & -1 & 1 & 1 & -1 & -1 & 1 & 1 & -1 & -1 & 1 & 1 & -1 & -1 & $\mathrm{y}_{14}$ \\
\hline-1 & 1 & -1 & 1 & -1 & 1 & -1 & 1 & -1 & 1 & -1 & 1 & -1 & 1 & -1 & $\mathrm{y}_{15}$ \\
\hline 1 & 1 & 1 & 1 & 1 & 1 & 1 & 1 & 1 & 1 & 1 & 1 & 1 & 1 & 1 & $\mathrm{y}_{16}$ \\
\hline
\end{tabular}

If columns 1, 2, 4 and 8 of Table 1 were used to set the level of four factors $A, B, C$ and $D$, respectively, then $\mathrm{y}_{1}$ through $\mathrm{y}_{16}$ represent the responses at the $2^{4}=16$ possible combinations of factor settings. This gives a $2^{4}=16$ run, two-level, four-factor, full factorial design. By using this $2^{4}$ full factorial design, the main effects of $A, B, C$ and $D$, as well as their interactions $A B, A C, A D, B C, B D, C D, A B C, A B D, A C D, B C D$ and $A B C D$ can be estimated. If it is required to study one more factor $E$ using the 16-run design, we have different choices. For design $\mathrm{d}_{1}$, the level of factor $E$ can be assign to column 15 . This gives a $2^{5-1}$ fractional factorial design. Since $15=1248$ or I $=2458$ where I denotes the column of +1 's, the estimate of the main effect $\mathrm{E}$ could not be separated from the effect of the interaction between $A, B, C$ and $D$. That is to say $E=A B C D$, or $I=A B C D E$. Here $I=2458$ is the defining relation or defining word of the $2^{5-1}$ design. The resolution of the design is 4 and the word-length pattern $W\left(\mathrm{~d}_{1}\right)=(0,0,0,1)$. For design $\mathrm{d}_{2}$, factor $E$ was assign to column $14=248$. The defining word is $I=128$ and the resolution is 3 . The word-length pattern $W\left(\mathrm{~d}_{2}\right)=(0,0,1,0)$. Obviously, $d_{1}$ is better than $d_{2}$ because it has higher resolution and minimum aberration. Both definition of resolution 
and minimum aberration are based on the hierarchical assumption that: (i) lower order interactions are more important than higher order interactions, (ii) effect of the same order are equally important. The advantage of $\mathrm{d}_{1}$ is obvious based on this principle because the main effect in $d_{1}$ is confounded with three-factor interactions and the main effect in $d_{2}$ is confounded with two-factor interactions. The 16 run $2^{\mathrm{m}-\mathrm{p}}$ designs can be used to study up to fifteen factors by assigning these factors to all fifteen columns. This design is denoted by $2^{15-11}$ and is called saturated design.

\section{Criteria for selecting blocked $2^{\mathrm{m}-\mathrm{p}}$ designs}

For unblocked $2^{\mathrm{m}-\mathrm{p}}$ designs, Ke and Tang [7] proposed a minimum $N$-aberration criterion to select designs by systematically minimizing the bias. The criterion was further studied and summarized by Cheng and Tang [3]. For further discussion about this issue see Tang and Deng [12], [13], Tang [10], [11] and Ke, et al [8]. Ke [14] proposed a design selection criterion as follows;

Design selection criterion: Let $N_{j,} j=2,3, \ldots, N_{m}$ be the number of $j$-factor interactions not in the model confounded with the effects in the postulated model including main effects, blocking effects, and some important two-factor interactions. We select optimal blocked $2^{\mathrm{m}-\mathrm{p}}$ designs by sequentially minimizing

$N_{2}, \ldots, N_{m}$.

To gain further insight in to the criterion, let's now examine the criterion in more detail. The postulated model consists of all main effects, important two-factor interactions, and blocking effects. Those two-factor interactions not in the model and other higher order interactions generally cause a bias on the estimation of the effects in the model. The measure of this bias as given by $N_{j}$, the number of $\mathrm{j}$-factor interactions outside the model that are confounded with the effects in the model. Under the hierarchical assumption that lower-order effects are more important than higher-order effects; then to minimize the bias, the confounding pattern of a design should be sequentially minimized. Hence this criterion selects optimal design that has minimum $N_{2}$, and so on

Suppose that five factors $A, B, C$, and $D$ and two-factor interactions $A D$ and $B C$ need to be studied by using a blocked design of 16 runs. Two designs $d_{\mathrm{I}}$ and $d_{2}$ were considered. Based on the provision of table 1 above, for $d_{1}$, the five treatment factors $A, B, C, D$ and $E$ were assigned to column $1,2,4,8$ and 15 and the blocking factor to column $11_{\mathrm{b}}$ ( ' $\mathrm{b}$ ' was used to indicate blocking factor). The interactions to be estimated should be 18 and 24 . Since $15=1248$ and $11_{b}=$ 128 , the defining contrast subgroup of the design is given by $\mathrm{I}=2458=128\left(11_{\mathrm{b}}\right)=145\left(11_{\mathrm{b}}\right)$. Hence we have $1=12458$, $2=458,4=258,8=245,15=1248,18=1245,24=58$ and $11 \mathrm{~b}=128=145$. Therefore $N_{2}=1, N_{3}=5$ and $N_{4}=2$. Note that the interactions between blocking factors and treatment are assumed not existent and are not counted here. The confounding pattern of $d_{1}$ is $(1,5,2)$ which means that one 2-factor interactions ,five 3 -factor interactions and two 4-factor interactions not included in the model are confounded with the effects in the model. For $d_{2}$, the five treatment factors $A, B, C, D$ and $E$ were assigned to column 1, 2, 4, 8 and 13 and the blocking factor to column $10_{\mathrm{b} .}$ The interactions to be estimated should be 18 and 24 . Since $13=148$ and $10_{\mathrm{b}}=28$, the defining contrast subgroup of the design $d_{2}$ is given by $\mathrm{I}=348=28\left(10_{\mathrm{b}}\right)=234\left(10_{\mathrm{b}}\right)$. Hence we have $1=1348,2=2348,4=38,8=34,13=148,18=134$, $24=238$ and $10_{\mathrm{b}}=28=234$. Hence the confounding pattern of $d_{2}$ is $(3,4,2)$ which means that three 2 -factor interactions, four 3-factor interactions, and two 4-factor interaction not included in the model are confounded with the effects in the model. Based on our design selection criterion, $d_{1}$ is better than $d_{2}$ because $N_{2}\left(d_{1}\right)<N_{2}\left(d_{2}\right)$.

\section{Evaluating the performance of the designs}

As stated earlier, the aim is to evaluate the performance of design with respect to its confounding pattern. In this regard, two designs were compared by estimating the bias accounted in the estimation of regression coefficient. The structure of the two designs compared is given in table 2, below.

Table 2: Structure of the two designs considered for this article

\begin{tabular}{cccccc}
\hline Design type & $\mathrm{M}_{\mathrm{t}}+\mathrm{M}_{\mathrm{b}}$ & Treatment factor & 2-factor interaction & Block factor & $\left(\mathrm{N}_{2}, \mathrm{~N}_{3}, \ldots . \mathrm{N}_{\mathrm{m}}\right)$ \\
\hline$D_{1}$ & $5+1$ & 7 & $(1,8)$ & 11 & $(0,6,1)$ \\
$D_{2}$ & $5+1$ & 3 & $(1,8)$ & 5 & $(4,2,2)$ \\
\hline
\end{tabular}

Design I denoted by $D_{1}$ is proposing that we assign the first four factors in the original column of the calculation matrix i.e. column 1, 2, 4 and 8 and confound the $5^{\text {th }}$ factor with column 7 and blocking factor with column 11 and estimate one 2 -factor interaction in column 9. While design II is proposing that we assign the first four factors in the original column of the calculation matrix i.e. column 1,2, 4 and 8 and confound the $5^{\text {th }}$ factor with column 3 and blocking factor with column 5 and estimate one 2 -factor interaction in column 9 . From table 2 the confounding pattern of $D_{1}$ is $(0,6,1)$ which means zero 2-factor interaction, six 3-factor interaction and one 4-factor interaction not in the model are confounded with the effect in the postulated model. For $D_{2}$ the confounding pattern of design II is $(4,2,2)$ which means four 2-factor interaction, two 3 -factor interaction and two 4 -factor interaction not in the model are confounded with the effect in the postulated model. The nature of the confounding pattern of a design determines the level of contamination which in turn lowers the precision of the parameter estimate. 


\subsection{Estimation of total bias}

The postulated model is given by

$Y=\alpha_{0} I+W_{1} \gamma_{1}+\varepsilon$

where $Y$ denotes the vector of $n$ observations, $\alpha_{0}$ is the grand mean, $I$ denotes the vector of $n$ ones, $\gamma_{1}$ is the vector of parameters containing all main effects, a set of important two-factor interactions, and blocking effects, $W_{1}$ is the corresponding design matrix, and $\varepsilon$ is the vector of uncorrelated random errors, assumed to have mean 0 and a constant variance, $\sigma^{2}$. Since other interactions among treatment factors may not be negligible the true model can be written as

$Y=\alpha_{0} I+W_{1} \gamma_{1}+X_{2} \alpha_{2}+X_{3} \alpha_{3}+\cdots+X_{m} \alpha_{m}$

From the postulated model in (1) above, the least square estimator,

$\hat{\gamma}=\left(W_{1}^{T} W_{1}\right)^{-1} W_{1}^{T} Y=n^{-1} W_{1}^{T} Y$.

To obtain the bias in the estimation of $\gamma$, the expectation of $\hat{\gamma}$ from the true model is used.

$E(\hat{\gamma})=\gamma_{1}+P_{2} \alpha_{2}+P_{3} \alpha_{3}+\cdots+P_{m} \alpha_{m}$, where

$P_{2}=n^{-1} W_{1}^{T} X_{2}$ and $P_{j}=n^{-1} W_{1}^{T} X_{j}$.

The bias of $\hat{\gamma}_{1}$ in estimating $\gamma_{1}$ is given by

$\operatorname{Bias}\left(\hat{\gamma}_{1}, \gamma_{1}\right)=P_{2} \alpha_{2}+P_{3} \alpha_{3}+\cdots+P_{m} \alpha_{m}$, where

$P_{2} \alpha_{2}$ Is the contribution of $\alpha_{2}$ to the bias, and $P_{j} \alpha_{j}$ is the contribution $\alpha_{j}$ to the bias. Since $\alpha_{j}$ is unknown we have to work with $P_{j}$. One size measure for matrix $P$ is given by $\|P\|^{2}=\operatorname{trace}\left(P^{T} P\right)$.

Recall that for design I the cofounding pattern is $(0,6,1)$ and $P_{j}=\left(W_{1}^{T} W_{1}\right)^{-1} W_{1}^{T} X_{j}$,

When $j=2$,

$P_{2}=\left(W_{1}^{T} W_{1}\right)^{-1} W_{1}^{T} X_{2}$. From the confounding pattern of design I, none of the two factor interaction not in the model confounded with the effect in the model. Therefore

$\left(\mathrm{W}_{1}^{\mathrm{T}} \mathrm{W}_{1}\right)^{-1}=\left(\begin{array}{llllllll}0.0625 & 0 & 0 & 0 & 0 & 0 & 0 \\ 0 & 0.0625 & 0 & 0 & 0 & 0 & 0 \\ 0 & 0 & 0.0625 & 0 & 0 & 0 & 0 \\ 0 & 0 & 0 & 0.0625 & 0 & 0 & 0 \\ 0 & 0 & 0 & 0 & 0.0625 & 0 & 0 \\ 0 & 0 & 0 & 0 & 0 & 0.0625 & 0 \\ 0 & 0 & 0 & 0 & 0 & 0 & 0.0625\end{array}\right)$
$W_{1}^{T} X_{2}=\left(\begin{array}{l}0 \\ 0 \\ 0 \\ 0 \\ 0 \\ 0 \\ 0\end{array}\right)$

$P_{2}=\left(W_{1}^{T} W_{1}\right)^{-1} W_{1}^{T} X_{2}=\left(\begin{array}{l}0 \\ 0 \\ 0 \\ 0 \\ 0 \\ 0 \\ 0\end{array}\right)$

$\operatorname{trace}\left(P_{2}^{T} P_{2}\right)=0$

When $j=3$, we have six 3-factor interaction not in the postulated model confounded with the effect in the model. The six 3-factor interactions are 247, 147, 127, 124, 128 and 478. 
$P_{3}=\left(W_{1}^{T} W_{1}\right)^{-1} W_{1}^{T} X_{3}=\left[\begin{array}{cccccc}1 & 0 & 0 & 0 & 0 & 0 \\ 0 & 1 & 0 & 0 & 0 & 0 \\ 0 & 0 & 1 & 0 & 0 & 0 \\ 0 & 0 & 0 & 0 & 0 & 0 \\ 0 & 0 & 0 & 1 & 0 & 0 \\ 0 & 0 & 0 & 0 & 0 & 0 \\ 0 & 0 & 0 & 0 & 1 & 1\end{array}\right]$

Trace $\left(P_{3}^{T} * P_{3}\right)=6$.

Similarly, when $j=4$, only one 4-factor interaction (2478) not in the postulated model confounded with the effect in the model.

$P_{4}=\left(W_{1}^{T} W_{1}\right)^{-1} W_{1}^{T} X_{4}=\left(\begin{array}{l}0 \\ 0 \\ 0 \\ 0 \\ 0 \\ 1 \\ 0\end{array}\right)$

Trace $\left(P_{4}^{T} * P_{4}\right)=1$

$\operatorname{Bias}\left(\hat{\gamma}_{1}, \gamma_{1}\right)=\operatorname{trace}\left(P_{2}{ }^{T} * P_{2}\right)+\operatorname{trace}\left(P_{3}{ }^{T} * P_{3}\right)+\operatorname{trace}\left(P_{4}{ }^{T} * P_{4}\right)=0+6+1=7$.

For design II the confounding pattern is $(4,2,2)$ and $P_{j}=\left(W_{1}^{T} W_{1}\right)^{-1} W_{1}^{T} X_{j}$.

When $j=2$

$\left(W_{1}^{T} W_{1}\right)^{-1}=\left[\begin{array}{llllllll}0.0625 & 0 & 0 & 0 & 0 & 0 & 0 \\ 0 & 0.0625 & 0 & 0 & 0 & 0 & 0 \\ 0 & 0 & 0.0625 & 0 & 0 & 0 & 0 \\ 0 & 0 & 0 & 0.0625 & 0 & 0 & 0 \\ 0 & 0 & 0 & 0 & 0.0625 & 0 & 0 \\ 0 & 0 & 0 & 0 & 0 & 0.0625 & 0 \\ 0 & 0 & 0 & 0 & 0 & 0 & 0.0625\end{array}\right]$

And

$\left(W_{1}^{T} X_{2}\right)=\left[\begin{array}{llll}16 & 0 & 0 & 0 \\ 0 & 16 & 0 & 0 \\ 0 & 0 & 0 & 0 \\ 0 & 0 & 0 & 0 \\ 0 & 0 & 16 & 0 \\ 0 & 0 & 0 & 0 \\ 0 & 0 & 0 & 16\end{array}\right]$ 
$P_{2}=\left(W_{1}^{T} W_{1}\right)^{-1} W_{1}^{T} X_{2}=\left[\begin{array}{cccc}1 & 0 & 0 & 0 \\ 0 & 1 & 0 & 0 \\ 0 & 0 & 0 & 0 \\ 0 & 0 & 0 & 0 \\ 0 & 0 & 1 & 0 \\ 0 & 0 & 0 & 0 \\ 0 & 0 & 0 & 1\end{array}\right]$

$\operatorname{Trace}\left(P_{2}{ }^{T} * P_{2}\right)=4$

When $j=3$

$P_{3}=\left(W_{1}^{T} W_{1}\right)^{-1} W_{1}^{T} X_{3}=\left[\begin{array}{cc}0 & 0 \\ 0 & 0 \\ 0 & 0 \\ 0 & 0 \\ 0 & 0 \\ 1 & 0 \\ 0 & 1\end{array}\right]$

$\operatorname{Trace}\left(P_{3}^{T} * P_{3}\right)=2$.

When $j=4$

Trace $\left(P_{4}^{T} * P_{4}\right)=2$.

$P_{4}=\left(W_{1}^{T} W_{1}\right)^{-1} W_{1}^{T} X_{4}=\left[\begin{array}{cc}0 & 0 \\ 0 & 0 \\ 1 & 0 \\ 0 & 1 \\ 0 & 0 \\ 0 & 0 \\ 0 & 0\end{array}\right]$

$\operatorname{Bias}\left(\hat{\gamma}_{1}, \gamma_{1}\right)=\operatorname{trace}\left(P_{2}{ }^{T} * P_{2}\right)+\operatorname{trace}\left(P_{3}{ }^{T} * P_{3}\right)+\operatorname{trace}\left(P_{4}{ }^{T} P_{4}\right)=4+2+2=8$.

\section{Conclusion}

Bias accounted In the estimation of $\gamma_{1}$ in the two designs were 7 and 8 respectively, this clearly shows the importance of taking in to consideration the nature of confounding pattern when selecting a design.

\section{References}

[1] Box, G.E.P. and Hunter, J.S. The $2^{\mathrm{k}-\mathrm{p}}$ fractional factorial designs, Technometrics, 3, (1961), 311-351.

[2] Chen, H. and Cheng, C.S. Theory of optimal blocking of $2^{\text {n-m }}$ design, The Annals of statistics, 27, (1999), 1948-1973.

[3] Cheng, C.S. and Tang, B. A general theory of minimum aberration and its applications, Annals of Statistics, 33, (2005), 944-958.

[4] Cheng, S.W. and Wu, C.F.J. Choice of optimal blocking schemes in two-level and three-level designs, Technometrics, 44, (2002), $269-277$.

[5] Franklin, M.F. Constructing tables of minimum aberration $\mathrm{P}^{\mathrm{n}-\mathrm{m}}$ designs, Technometrics, 26, (1984), $225-232$.

[6] Fries, A. and Hunter, W.G. Minimum aberration $2^{\mathrm{k}-\mathrm{p}}$ designs, Technometrics, 22, (1980), 601-608.

[7] Ke, W. and Tang B. Selecting $2^{\mathrm{m}-\mathrm{p}}$ designs using a minimum aberration criterion when some two-factor interactions are important, Technometrics, 45, (2003), 352-360.

[8] Ke, W., Tang, B., and Wu, H. Compromise plans with clear two-factor interactions, Statistica Sinica, 15, (2005), 709-715.

[9] Sitter, R.R., Cheng, J., and Feder, M. fractional resolution and minimum aberration in blocked $2^{\text {n-k }}$ designs, Technometrics 39, (1997), 382390

[10] Tang, B. Theory of J-characteristics for fractional factorial design and projection justification of minimum $\mathrm{G}_{2}$ aberration, Biometrika, 88, (2001), 401-407.

[11] Tang, B. Orthogonal arrays robust to non-negligible two-factor interactions, Biometrika, 93, (2006), 137-146

[12] Tang, B., and Deng, L.Y. Minimum G G-aberration for non-regular fractional factorial designs. Annals of Statistics, 27, (1999), $1914-1926$. 
[13] Tang, B., and Deng, L.Y. Construction of generalized minimum aberration design of 3, 4, and 5 factors, J. of Stat. Planning and inference, $113,(2003), 335-340$.

[14] Ke, W., Optimal selection of blocked two-level fractional factorial designs, Applied Mathematical Sciences, 1, (2007), 1069-1082

[15] Xu, H. Blocked regular fractional factorial designs with minimum aberration, The Annals of Statistics, 34, (2006), $2534-2553$.

[16] Xu, H., and Lau, S. Minimum aberration blocking schemes for two and three-level fractional factorial designs, Journal of statistical planning and inference, 136, (2006), 4088-4118.

[17] Xu, H., and Mee, R. W. (2010) Minimum aberration blocking schemes for 128-Run Designs, Journal of statistical planning and inference, 140, 3213-3229 